\title{
Soft-packaged sensory glove system for human-like natural interaction and control of prosthetic hands
}

\author{
Min Ku Kim (1) ', Ramviyas Nattanmai Parasuraman ${ }^{2,3}$, Liu Wang ${ }^{4}{ }^{4}$, Yeonsoo Park ${ }^{5}$, Bongjoong Kim5 ${ }^{5}$ Seung Jun Lee ${ }^{5}$, \\ Nanshu Lu4, Byung-Cheol Min ${ }^{2}$ and Chi Hwan Lee ${ }^{1,5,6}$
}

\begin{abstract}
People with hand amputations experience strenuous daily life challenges, often leading to lifelong use of a prosthetic hand(s) and services. Modern advanced prosthetic hands must be able to provide human hand-like sensory perceptions to receive external stimuli during daily activities while simultaneously replicating a realistic appearance and physical properties to naturally integrate in social contexts; however, the practical realization of these issues are impeded by a lack of effective methodologies. Herein, we present an optimal set of materials, design layouts, and fabrication schemes to construct an easy-to-wear seamless electronic glove (e-glove) suitable for arbitrary hand shapes that provides all of the desired human hand-like features. The system configuration involves a connection to a control wristwatch unit for real-time display of sensory data measured and remote transmission to the user. The experimental and computational studies provide details regarding the underlying principles of the materials selection, mechanics design, and operational management of the entire system. The demonstration of the e-glove system in interactions with human subjects illustrates the utility, comfort, and convenience of this device.
\end{abstract}

\section{Introduction}

The human hand is one of the foremost parts of the body and serves as a versatile physical instrument for daily and social activities. Any disfigurement of this powerful physical instrument can affect a person's quality of life due to reduced manual dexterity and sensory reception and an unnatural appearance ${ }^{1,2}$. As a result, nearly $50 \%$ of all people with hand amputations require psychological intervention for isolation accompanied by depression, fatigue, anxiety, or even suicidal ideation ${ }^{2,3}$. Current evidence-based treatments rely on the use of the prosthetic

\footnotetext{
Correspondence: Nanshu Lu (nanshulu@utexas.edu) or Byung-

Cheol Min (minb@purdue.edu) or Chi Hwan Lee (lee2270@purdue.edu)

${ }^{1}$ Weldon School of Biomedical Engineering, Purdue University, West Lafayette, IN 47907, USA

${ }^{2}$ Department of Computer and Information Technology, Purdue University, West Lafayette, IN 47907, USA

Full list of author information is available at the end of the article.

These authors contributed equally: Min Ku Kim, Ramviyas Nattanmai

Parasuraman, Liu Wang
}

hand(s) to restore vital mobility that is important in many daily and social interactions, such as gripping, handshakes, gentle stroking, and petting ${ }^{4,5}$. The recent development of flexible and stretchable materials and electronics provides both mechanical softness and sensory perception to detect changes in external stimuli including pressure, temperature, and hydration ${ }^{6-11}$. However, the seamless integration of these materials around prosthetic hands is still challenging due to geometric complexity, resulting in continued disfigurement and poor physical coupling. The ability to directly assemble flexible and stretchable electronic circuit materials and devices on a commercial stretchable glove could provide an easy-towear seamless platform suitable for arbitrary prosthetic hands ${ }^{12-14}$.

Herein, we present a set of advanced materials, design layouts, and fabrication schemes for the realization of an electronic sensory glove (e-glove) system that is directly built on a commercial stretchable nitrile glove, allowing 
seamless fit on arbitrary prosthetic hands through the intrinsic ergonomic design of the glove for any hand shapes and sizes within the regular range of adult hand sizes. The e-glove system is configured with flexible and stretchable forms of multimodal sensors to collect various types of information, such as pressure, temperature, and moisture while simultaneously offering realistic human hand-like appearance, softness, and warmth. The capabilities of the real-time display of the sensory data measured on a control wristwatch unit and remote transmission to an external reader for data postprocessing provide benefits and convenience to the user. The fabrication of the e-glove system involves a cost-effective hybrid printing technology that combines screen-printing and transfer-printing methods and is tailored to laminate multiple layers of electronic circuit materials on a commercial stretchable glove. Both the experimental and computational investigations reveal key features of the underlying materials and structures and the mechanics aspects of the design variables. The results demonstrate the utility of the e-glove system during the interactions and control of a prosthetic hand with objects and humans in many daily and social settings.

\section{Materials and methods}

\section{Finite element analysis (FEA)}

The commercial software ABAQUS (standard 6.13) was used to study the mechanical behavior of the prototype devices under stretching, bending and folding. A multilayered model was built, i.e., an encapsulated $\left(h_{\text {encap }}=\right.$ $500 \mu \mathrm{m})$-PDMS/Ag $\left(h_{\mathrm{PDMS}}=80 \mu \mathrm{m}, h_{A g}=45 \mu \mathrm{m}\right)$-ероху adhesive $\left(h_{\text {epoxy }}=70 \mu \mathrm{m}\right)$-nitrile glove $\left(h_{\text {PDMS }}=150 \mu \mathrm{m}\right)$ composite. Since a serpentine-shaped $\mathrm{Ag}$ trace was embedded in the PDMS, a partition of exactly the same shape as the serpentine layout was created with effective mechanical properties, e.g., Young's modulus:

$$
E_{\mathrm{eff}}=E_{\mathrm{Ag}}\left(\frac{h_{\mathrm{Ag}}}{h_{\mathrm{PDMS}}+h_{\mathrm{Ag}}}\right)+E_{\mathrm{PDMS}}\left(\frac{h_{\mathrm{PDMS}}}{h_{\mathrm{PDMS}}+h_{\mathrm{Ag}}}\right)
$$

where $E_{\mathrm{Ag}} \approx 0.2 \mathrm{MPa}$ (obtained from the vendor) and $E_{\mathrm{PSMS}} \approx 2.5 \mathrm{MPa}$. For the epoxy adhesive layer and glove, $E_{\text {epoxy }} \approx 3.38 \mathrm{MPa}$ (urethane based), and $E_{\text {glove }} \approx 21 \mathrm{MPa}$ (from the product sheet). Because the thickness of each layer $(\sim \mathrm{mm})$ was substantially smaller than the lateral dimensions of the device $(\sim \mathrm{cm})$, the device was modeled as a composite shell with element S4R. Incompressible neo-Hookean constitutive behavior was assigned to all the layers.

\section{Thermal analysis}

COMSOL 5.3 electric currents and heat transfer modules were used for the time-dependent joule-heating simulation. The heat was generated when an electric current of 0.24 A was applied to the serpentine-shaped Ag traces. The heating time was $120 \mathrm{~s}$. The thermal properties of the materials were obtained from the vendor product sheets and https://thermtest.com/materialsdatabase.

\section{Fabrication of the e-glove system}

The fabrication began with a commercial stretchable nitrile glove (Kimberly-Clark, Irving, TX, USA) by gluing an epoxy (Loctite 4902, Henkel, Rocky Hill, CT, USA) over the surface to provide adhesive support, followed by curing in an oven at $70^{\circ} \mathrm{C}$ for $10 \mathrm{~min}$. A conductive Ag ink (125-19FS, Creative Materials, Ayer, MA, USA) was screen-printed on the surface of the glove to define the electrical interconnectors through a mesh screen (Ryonet, Vancouver, WA, USA) featured with photolithographicpatterned filamentary serpentine traces. Active sensing elements were then delivered from the donor substrate to the desired locations of the glove in a spatially distributed manner by using sequential transfer printing operations. Subsequently, the glove was annealed in an oven at $70^{\circ} \mathrm{C}$ for $2 \mathrm{~h}$ to secure the bonding between the sensing elements and the conductive Ag ink. The entire structure was dip-coated in an uncured silicone elastomer (Ecoflex $^{\mathrm{TM}}$, Smooth-On, Macungie, PA, USA) to form a thin sealing layer on the surface, followed by complete curing at $70^{\circ} \mathrm{C}$ for $30 \mathrm{~min}$. The steps of transfer printing and dipcoating were iterated to stack multiple layers consisting of different types of sensors. A subsequent dip-coating of the entire structure with a silicone elastomer (Dragon Skin Series, Smooth-On) to form the outermost skin layer completed the fabrication.

\section{Incorporation of human hand-like appearance into the skin layer}

The process began by gently pouring a solution of silicone elastomer (Body Double ${ }^{\mathrm{TM}}$, Smooth-On) on a volunteer's hand to generate a custom-fitted mold. An agent, a 1:4 mixture of petroleum jelly and mineral spirits, was applied to the interior surface of the mold for an easy and clean release. A mixture of silicone elastomer (Dragon Skin Series, Smooth-On, USA) and skin-tone colorant (Slig-Pig ${ }^{\mathrm{TM}}$, Smooth-On, USA) was thinly applied to the interior surface of the mold. The aforementioned asprepared e-glove was placed inside the mold and then annealed at room temperature for $1 \mathrm{~h}$. The release of the cured structure from the mold completed the process.

\section{Fabrication of control wristwatch unit}

The fabrication began by printing a wristwatch case and buttons by using 3D printing equipment (Fortus $400 \mathrm{mc}$, Stratasys, Eden Prairie, MN, USA) with a fused deposition of ABS (Acrylonitrile butadiene styrene) plastics. The internal printed circuit board (PCB) was fabricated on a 
$\mathrm{Cu} / \mathrm{PI}$ film $(12 \mu \mathrm{m} / 12 \mu \mathrm{m}$ thick, Pyralux AP121200EM, DuPont, Durham, NC USA). A dry film photoresist (Riston MM540, DuPont, USA) was applied to the $\mathrm{Cu} / \mathrm{PI}$ film by using a hot roller laminator (AL13P, Apache Laminator, USA), followed by photolithographic patterning and a wet etching process (CE-100, Transene Company, Danvers, MA, USA) to form the solder pads and traces. The resulting metal patterns were electroplated with a layer of tin ( $\mathrm{Sn}$ ) (Bright Electroless Tin, Transene, USA) for oxidation protection. Other necessary electronic components such as a microcontroller unit, multiplexer, and switches were soldered on the PCB and assembled inside the $3 \mathrm{D}$ printed wristwatch case along with an organic light-emitting diode (OLED) display (1673, Adafruit Industries, New York, NY, USA). The rigid housing case was thoroughly sealed with insulating tape (Kapton) to reduce the risk of potential short circuits. A summary of the electronic components used in this unit appears in Supplementary Table S1. Finally, the entire structure was mounted on a commercial wristband by using an epoxy adhesive.

\section{Fabrication of artificial hand}

The fabrication began with fused deposition of $A B S$ plastics using 3D printing equipment (Fortus $400 \mathrm{mc}$, Stratasys, USA) to print 15 parts consisting of both the finger and palm sections. The printed parts were assembled using cyanoacrylate adhesive and neoprene rubber sheets ( $2 \mathrm{~mm}$ thick) to form the basic structural frame of a hand. Each joint of the artificial hand was connected with fishing lines (Sufix 832, Rapala VMC, Minnetonka, MN, USA), allowing movement of the fingers and thumb by external adjustment of the line tension.

\section{Recording of pressure and temperature}

The arrays of pressure and temperature sensors were configured in the same way. Custom-miniaturized constant current preamplifier circuits consisting of op-amp (TLV333, Texas Instruments, Dallas, TX, USA) and bipolar junction transistors (BJT, MMBT3906SL, ON Semiconductor, Phoenix, AZ, USA) were used to supply a constant current of $100 \mu \mathrm{A}$ to the sensors. Multiplexers with 32 channels (ADG726, Analog Devices, Norwood, MA, USA) controlled by a microcontroller unit (RFD77101, RF Digital, Hermosa Beach, CA, USA) were used to switch between the sensors while the voltage drop was measured across the sensing elements during recording. The changes in the voltage corresponding to external stimuli of pressure and temperature were measured by a 16-bit resolution analog-to-digital converter. The data measured were displayed on the control wristwatch unit and simultaneously transmitted to an external reader such as a commercial smartphone or tablet via Bluetooth communication.

\section{Recording of hydration}

A capacitive hydration sensor with interdigitated microelectrode arrays was assembled on the index fingertip of the e-glove. To facilitate the direct contact of the embedded hydration sensor with environmental moisture, a $3 \times 3$ array of small openings ( $1 \mathrm{~mm}$ each in diameter) were punched through the outermost skin layer on the fingertip. A capacitance-to-digital converter (FDC1004, Texas Instruments, USA) was used in conjunction with a microcontroller unit (RFD77101, RF digital, USA) to measure the capacitance during recording. The data were processed by the microcontroller unit and then displayed on the control wristwatch unit and remotely transmitted to an external reader such as a commercial smartphone or tablet.

\section{Fabrication of networked Ag nanowire-mesh}

The fabrication began by synthesizing $\mathrm{Ag}$ nanowires with a mixture of $50 \mathrm{~mL}$ of ethylene glycol (EG, 9300-03, J.T.Baker, Radnor, PA, USA), $400 \mu \mathrm{L}$ of copper (II) chloride $\left(\mathrm{CuCl}_{2}, 4 \mathrm{mM}, 487847\right.$, Sigma-Aldrich, St. Louis, $\mathrm{MO}, \mathrm{USA}$ ), and $15 \mathrm{~mL}$ of polyvinylpyrrolidone (PVP, 0.147 M, 856568, Sigma-Aldrich, USA) in a preheated oil bath (CG-1100, Chemglass, Vineland, NJ, USA) at $150^{\circ} \mathrm{C}$ for $1 \mathrm{~h}^{15}$. Approximately $15 \mathrm{~mL}$ of $\mathrm{Ag}$ nitrate $\left(\mathrm{AgNO}_{3}\right.$, $0.094 \mathrm{M}$ ) was injected at a rate of $0.5 \mathrm{~mL} / \mathrm{min}$ by using a syringe pump (AL-4000, World Precision Instruments, Sarasota, FL, USA) until the color of the solution changed from ivory to grey. This step was repeated $3 \sim 5$ times while adding $10 \mathrm{~mL}$ of the mixed precursor solution in each step. The resulting solution was cooled to room temperature, followed the addition of $450 \mathrm{~mL}$ of acetone. The mixture was then centrifuged to extract the Ag nanowires from the mixture. The as-prepared $\mathrm{Ag}$ nanowires were filtered through a Teflon filter $(0.2 \mu \mathrm{m}$ pore size, Sterlitech, Kent, WA, USA) by using a vacuum-assisted Buchner funnel (1960-054, Whatman, UK) to form a sheet of highly networked Ag nanowire-mesh.

\section{Fabrication of electrophysiological recording electrodes}

The aforementioned as-prepared networked Ag nanowire-mesh was placed on a Si wafer coated with bilayers of poly(methyl methacrylate) (PMMA, $1 \mu \mathrm{m})$ and PI $(1 \mu \mathrm{m})$ on the surface, followed with standard photolithography and reactive-ion-etching (RIE) etching to define the necessary patterns in filamentary serpentine traces for the electrophysiological (EP) electrodes. The resulting structure was immersed in a solution of acetone for $\sim 1 \mathrm{~h}$ to dissolve the PMMA layer, allowing the EP electrodes to release from the Si wafer to subsequently be installed in the e-glove.

\section{Recording of EP signals}

The EP recording electrodes were installed around the tip of the thumb of the e-glove. Then, the EP electrodes 
were attached to the skin of the chest and forearm of a volunteer (age: 30) during the recording of electrocardiogram (ECG) and electromyogram (EMG) signals, respectively. The signals measured were remotely sent to an external computing system by using a portable wireless unit (BioRadio ${ }^{\mathrm{TM}}$, Great Lakes NeuroTechnologies, Cleveland, OH, USA) that was connected to the e-glove. Commercial software (BioCapture, Great Lakes NeuroTechnologies, USA) was used with filtering at $60 \mathrm{~Hz}$ and bandpass at $0.5 \sim 100 \mathrm{~Hz}$ (ECG) and $10 \sim 500 \mathrm{~Hz}$ (EMG). The filtered data were remotely exported to MATLAB for data postprocessing.

\section{Studies on human subjects}

All the studies on human subjects were approved by the Purdue Institutional Review Board (protocol \#: 1711019949) and conducted in compliance with the applicable regulations.

\section{Results}

Figure 1a shows a series of optical images of a representative e-glove platform that contains multiple stacked arrays of sensor elements (insets), including (1) a total of 20 pressure sensors $(6.5 \mathrm{~mm} \times 4.6 \mathrm{~mm}$ each) evenly distributed over the entire area, (2) a capacitive-based moisture sensor $(7 \mathrm{~mm} \times 6.3 \mathrm{~mm})$ on the index fingertip, and (3) 16 resistive-based temperature sensors $(1 \mathrm{~mm} \times$ $1 \mathrm{~mm}$ each) at the center of the palm. The representative electrical characteristics of the embedded sensor elements as a function of the externally applied stimuli are summarized in Fig. 1b. The results indicate that the sensitivities of the pressure, temperature and moisture sensors are $\sim 10 \mu \mathrm{A} / \mathrm{kPa}, \sim 1 \mathrm{pF} / 20 \mu \mathrm{L}$ moisture level, and $\sim 0.6 \mathrm{mV} /{ }^{\circ} \mathrm{C}$ within the ranges of the applied pressure of $0 \sim 200 \mathrm{kPa}$, moisture of $0 \sim 100 \%$, and temperature of $20 \sim 50{ }^{\circ} \mathrm{C}$, respectively, without suffering any degradation in performance. The fabrication begins by gluing a thin layer of a flexible epoxy (Loctite 4902, Henkel, USA) on the surface of a commercial stretchable nitrile glove (Kimberly-Clark, USA) to serve as an adhesive. Subsequent screen-printing of a flexible Ag paste (125-19FS, Creative Materials, USA, $\sim 0.05 \Omega / \mathrm{sq} / \mathrm{mil}$ ) configured with a fractal serpentine layout (inset image) defines a stretchable form of conducting interconnectors. The employment of a pick-and-place transfer printing method results in the delivery of active sensor elements to predefined locations in an array layout that meets the spatial resolution requirements ${ }^{16-18}$. Dip-coating of the entire structure in an uncured silicone elastomer $\left(\mathrm{Ecoflex}^{\mathrm{TM}}\right.$, Smooth-On, USA), followed by complete curing at $70^{\circ} \mathrm{C}$ for $\sim 30 \mathrm{~min}$, leads to a thin sealing layer $(\sim 300 \mu \mathrm{m}$ thick) over the surface to serve as electrical insulation for the subsequent layer. These steps can be iterated to provide stacked layers of sensor arrays for multimodal sensing capabilities. Finally, lamination of another thin sealing layer $(\sim 300 \mu \mathrm{m}$ thick) with a silicone elastomer (Dragon Skin Series, Smooth-On, USA) forms the outermost skin layer not only to provide human skin-like mechanical softness and resilience but also to ensure the mechanical integrity and reduce any potential risk of interfacial delamination ${ }^{19}$. The details of the assembly procedures appear in the Methods section.

The abilities to provide a real-time display of the sensory data measured and to remotely transmit the data to an external reader (e.g., commercial smartphone or tablet) for data postprocessing can improve the workflow between the e-glove system and the user, thereby offering operational efficiency and convenience. Figure 1c shows a custom-built control wristwatch unit that is wired to the e-glove system via a flexible anisotropic conductive film (ACF) cable (HST-9805-210, Elform, Fallon, NV, USA). The enlarged image highlights the organic light-emitting diode (OLED) display for the display of information, user interface navigation, and operational switches for function setting and control. The internal circuitry (Fig. 1d) of the control wristwatch unit includes (1) a 32-bit ARM Cortex M0 processor-based microcontroller (RFD77101, RF Digital, USA, $10 \times 7 \times 2.5 \mathrm{~mm}$ ) for data collection and wireless transmission via Bluetooth ${ }^{\mathrm{TM}}$, (2) a rechargeable battery $(3.6 \times 2.0 \times 0.56 \mathrm{~cm}, 350 \mathrm{mAh})$ for a power source, (3) a differential amplifier (INA333, Texas Instruments, USA) for front end detection and amplification of electrical signals, and (4) a 3D-printed package made of ABS plastics for housing. The overall workflow diagram of the embedded circuits appears in Supplementary Fig. S1 with more details in the Methods section. The use of the wristwatch allows the provision of immediate feedback to the prosthetic user through visual cues, which can provide two-dimensional data perception/visualization customizable to individual needs.

Prosthetic hands encounter many complex operations in daily and social activities including shaking a hand, tapping or punching an object, and holding hot/cold and dry/wet surfaces ${ }^{4}$. Given these circumstances, the realtime detection of pressure, temperature, and hydration from a prosthetic hand can provide useful information to the user. To illustrate this possibility, representative uses of the e-glove system in several daily circumstances envisioned are demonstrated by using a 3D-printed artificial hand as a surrogate for a prosthetic hand (Supplementary Fig. S2 \& Movie S1). Figure 2a shows an optical image of the e-glove grasping a baseball; the monitoring of the pressure exerted across the whole palm area is carried out by an array of 20 pressure sensors. The inset image shows an embedded single sensor element that includes a pressure-sensitive polymer (Velostat ${ }^{\mathrm{tm}}, 3 \mathrm{M}$, Maplewood, MN USA). Figure $2 \mathrm{~b}$ presents the results of postprocessed data, revealing detailed visual information about how 

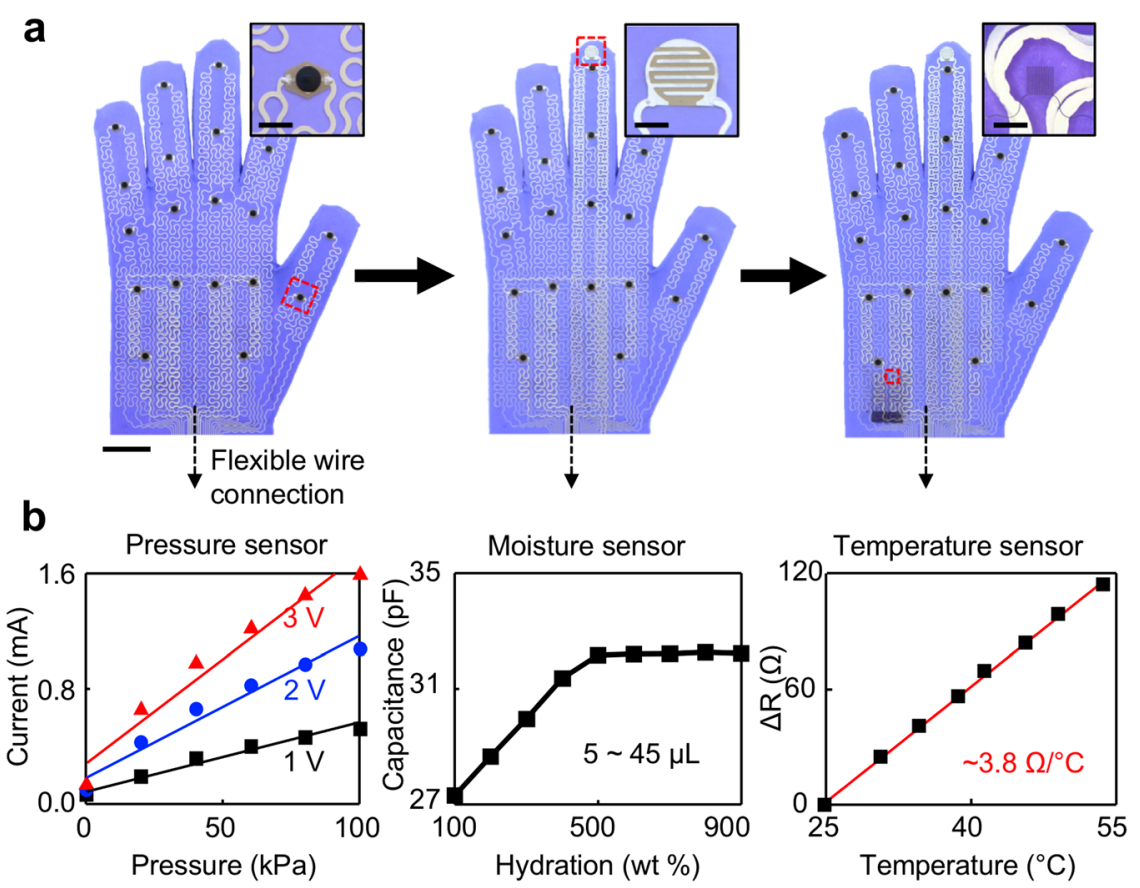

C
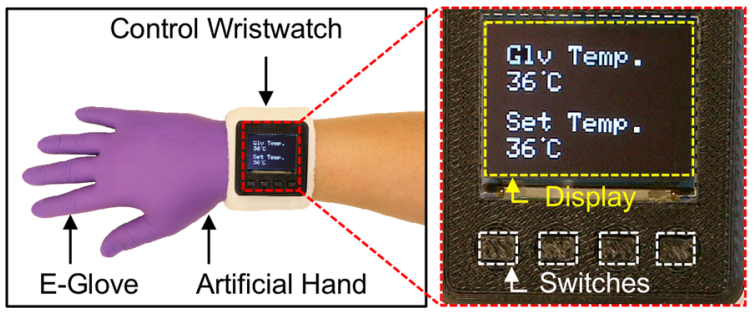

d

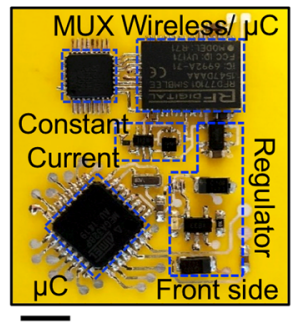

Fig. 1 Basic layouts and configurations of the e-glove system. a A series of optical images for a representative e-glove platform that contains multiple stacked arrays of sensor elements including pressure (left), moisture (middle), and temperature (right) sensors. Scale bar is $25 \mathrm{~mm}$. The inset images show an enlarged view of the embedded sensor elements. Scale bars are $4 \mathrm{~mm}$ (left), $3 \mathrm{~mm}$ (middle) and $1 \mathrm{~mm}$ (right), respectively. b Representative electrical characteristics of the embedded sensor elements as a function of externally applied stimuli. c Optical images of a custombuilt wristwatch unit connected to the e-glove system. Scale bars are $6 \mathrm{~cm}$ (left) and $1 \mathrm{~cm}$ (right), respectively. $\mathbf{d}$ Optical image of the embedded internal circuitry in the wristwatch unit. Scale bar is $5 \mathrm{~mm}$

hard/easy the prosthetic hand holds the baseball in a spatially resolved manner. Representative results of the electrical characteristics of the embedded sensor element appear in Fig. 2c, exhibiting a sensitivity of $\sim 4 \mu \mathrm{S} / \mathrm{kPa}$. The results indicate that the e-glove system is capable of distinguishing delicate changes in pressure that a human hand might experience in daily activity with a dynamic range (linear response) up to $\sim 100 \mathrm{kPa}$. The effects of different skin layer thickness $(100-500 \mu \mathrm{m})$ and variations in environmental temperature $\left(30-50^{\circ} \mathrm{C}\right)$ on the sensing performance appear in Supplementary Fig. S3a. The experimental results characterizing the repeatability and reliability of the sensor under cyclic loading at different levels of applied pressure are summarized in Supplementary Fig. S3b.
Another important sensory function for replicating human hand-like perception is the ability to detect moisture and temperature ${ }^{7}$. Figure $2 \mathrm{~d}$ provides an example for the use of the e-glove system to identify the dampness of a wet diaper by using an embedded capacitive hydration sensor positioned around the fingertip. Representative measurement results appear in Fig. 2e, indicating that an abrupt increase of the capacitance occurs when the e-glove touches a wet area of the diaper. A separate control measurement using a commercial moisture sensor (SEN-13322, SparkFun Electronics, Niwot, CO, USA) provides consistent results (Fig. 2f). The change in the capacitance over time for different levels of moisture appears in Supplementary Fig. S4. The use of the e-glove system to detect the temperature on the surface of 

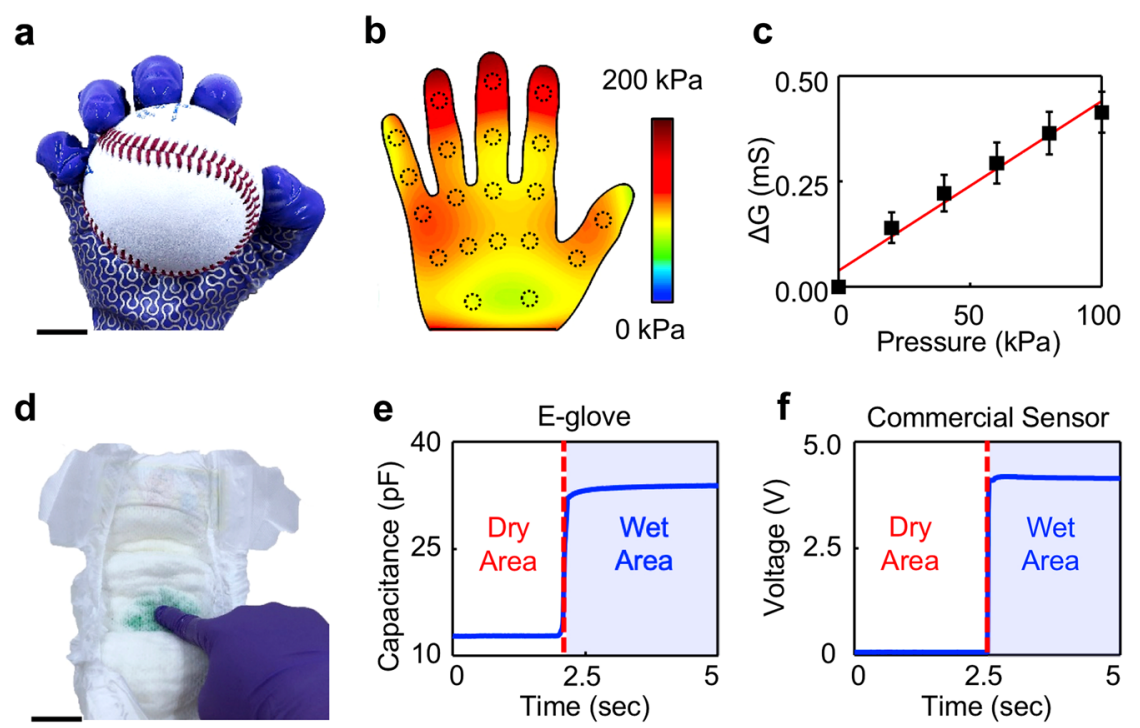

g

h
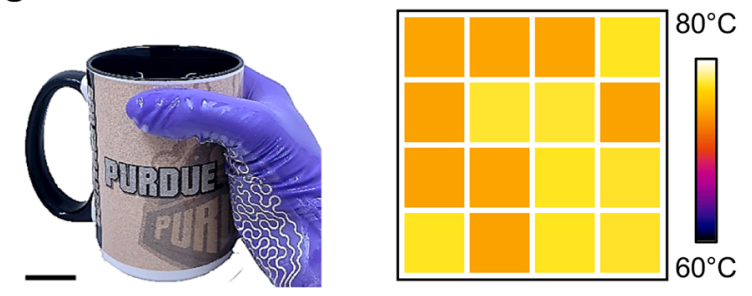

i
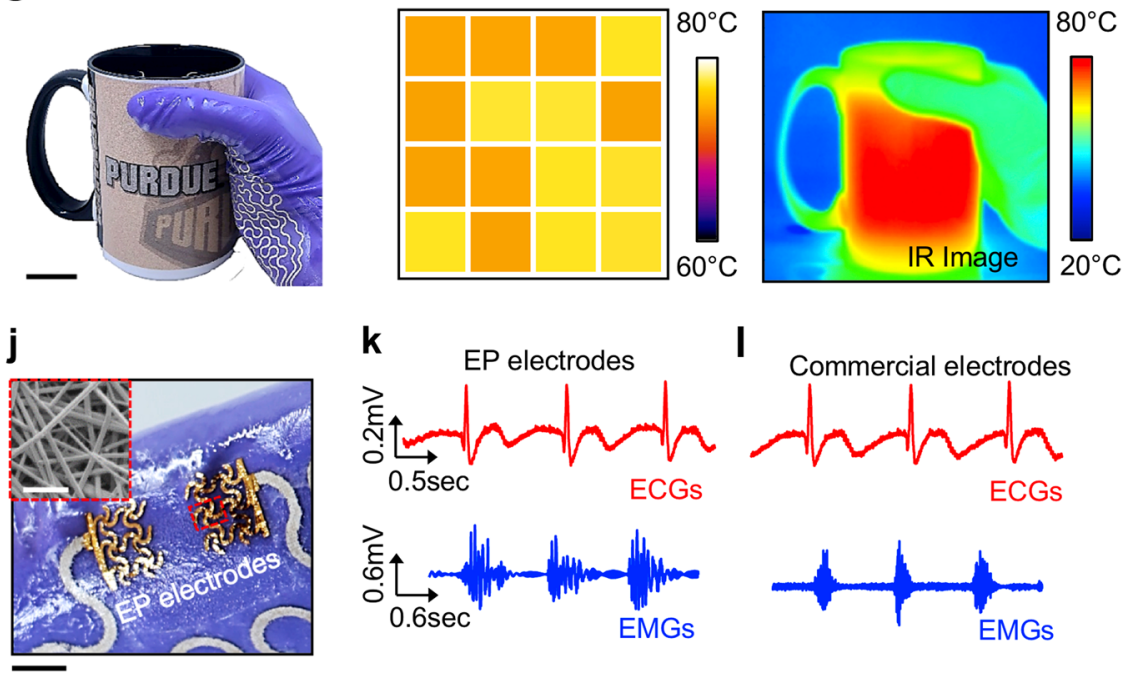

I Commercial electrodes
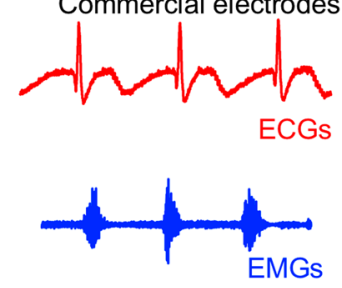

Fig. 2 Demonstration of human hand-like multimodal perception. a Optical image of the e-glove system grasping a baseball. Scale bar is $25 \mathrm{~mm}$. b Results of the recording of pressure. $\mathbf{c}$ Change of conductance as a function of pressure applied for the embedded single sensor element. $\mathbf{d}$ Optical image of the e-glove system touching a wet diaper. Scale bar is $5 \mathrm{~cm}$. e Results of the recording of hydration. $\mathbf{f}$ Results of control measurements by using a commercial hydration sensor. $\mathbf{g}$ Optical image of the e-glove system holding a cup of hot water. Scale bar is $5 \mathrm{~cm}$. $\mathbf{h}$ Results of the recording of temperature. $\mathbf{i}$ Results of control measurements by using a commercial infrared (IR) sensor. $\mathbf{j}$ Optical image of

electrophysiological (EP) electrodes installed around the thumb of the e-glove system. The inset SEM image highlights the embedded networked Ag nanowire-mesh. Scale bars are $4 \mathrm{~mm}$ and $600 \mathrm{~nm}$ (inset), respectively. $\mathbf{k}$ ECG (top) and EMG (bottom) results measured from the human skin. I Control measurement results from commercial EP recording electrodes

a cup containing hot water $\left(\sim 80^{\circ} \mathrm{C}\right)$ appears in Fig. $2 \mathrm{~g}$. The embedded sensor positioned on the palm area contains a $4 \times 4$ array of temperature sensors made of $\mathrm{Au}$ $(100 \mathrm{~nm}$ thick) and filamentary serpentine interconnectors ( $\mathrm{Au}, 300 \mathrm{~nm}$ thick). Figure $2 \mathrm{~h}$ presents the measurement results of the spatial temperature distribution when the e-glove system remains in contact with the cup. For a control comparison, real-time, simultaneous monitoring of the temperature occurs by using a commercial infrared (IR) camera (FLIR SC645, sensitivity: $0.05^{\circ} \mathrm{C}$ ) to confirm the surface temperature (Fig. 2i). In these demonstrations, the data measured are displayed on the screen of a control wristwatch unit (single point monitoring) and wirelessly transferred to an external reader such as a smartphone (multiple array monitoring), as appearing in Supplementary Fig. S5. The corresponding 
power consumption and estimated operation time for these sensor elements are summarized in Supplementary Table S2.

Another interesting aspect arises from the versatility of the e-glove system to provide extended capabilities beyond human sensory perception; i.e., to identify heart rates for on-demand access to health care and to monitor muscle fatigue during/after sport and exercise ${ }^{20}$. Fig. $2 \mathrm{j}$ shows an experimental demonstration that involves the use of the e-glove system for recording the electrical activities of the heart and muscles, such as ECGs and eEMGs, via the human skin. A separate prototype device consisting of an EP sensor on the outermost surface of the tip of the thumb is demonstrated by a using highly networked Ag nanowire-mesh (inset) patterned in a standard two-electrode configuration to serve as the EP electrodes. The use of a networked Ag nanowire-mesh offers useful features that enable high-fidelity coupling between the EP electrodes and the human skin against various loading conditions such as stretching and scratching ${ }^{21}$. The measurement results in Fig. 2k demonstrate the high-level recording of ECGs (top) and EMGs (bottom) while the EP electrodes remain in direct contact on the chest and the forearm, respectively (Supplementary Fig. S6). The ECGs and EMGs measured demonstrate clear detection of the P, $\mathrm{Q}, \mathrm{R}, \mathrm{S}$, and $\mathrm{T}$ waves and electrical currents generated in the muscles during contraction (neuromuscular activities), respectively. These recordings are qualitatively comparable with those obtained using commercial EP recording electrodes (RedDot ${ }^{\mathrm{TM}}, 3 \mathrm{M}$, USA) (Fig. 2l). The details of the EP measurements appear in the Methods section.

Human skin is elastic, flexible, and stretchable. Accordingly, the e-glove system demands the corresponding physical properties without any degradation in the performance of the embedded electronic materials. To achieve these physical properties, several strategies are used as follows: (1) the outermost skin layer of the e-glove system is comprised of a thin layer $(\sim 300 \mu \mathrm{m}$ thick $)$ of a silicone elastomer (Young's modulus $(E) \approx 0.5 \mathrm{MPa}$ ) that can provide softness and resilience similar to those of adult human skin ${ }^{19}$, (2) the constituent materials of the eglove system (e.g., a nitrile glove for the substrate, flexible Ag paste for interconnectors, and silicone elastomers for insulation/encapsulation) are flexible to accommodate mechanical loads during use and fitting, and (3) the filamentary serpentine traces incorporated along the electrical interconnectors provide the ability to mechanically isolate embedded semiflexible and rigid electronic components (e.g., capacitive hydration and temperature sensors) against stretching ${ }^{22}$.

Figure 3a (top) shows a representative optical image of a unit filamentary serpentine trace of the flexible Ag paste on a nitrile glove under stretching at $40 \%$, displaying no visible defects. The results of the finite element analysis (FEA) in Fig. 3a (bottom) reveal the maximum principal strains ( $\varepsilon \sim 33 \%)$ of the constituent material (i.e., Ag paste). Representative images of the damaged units with different geometries after stretching beyond the fracture limit (50 100\%) appear in Supplementary Fig. S7. The corresponding FEA results under different stretching conditions and by using a testbed unit embedded with a rigid sensor element are summarized in Supplementary Fig. S8. The experimental and computational (FEA) results of the testbed unit under bending (Fig. 3b) and folding (Fig. 3c) produce consistent results. Figure $3 \mathrm{~d}$ shows the measurement results of the relative resistance $\left(R / R_{0}\right)$ of the testbed unit under stretching up to $40 \%$ (left), bending/ folding (middle), and twisting up to $180^{\circ}$ (right). The results confirm that the $R / R_{0}$ barely changes within less than $\sim 5 \%$ for the mechanical deformations and then completely recovers when released. These results are consistent against repeated cycles of folding, while the R/ $R_{0}$ increases up to $\sim 2$ and $\sim 3$ against 2000 cycles of stretching at 30 and $60 \%$ strains, respectively (Supplementary Fig. S9).

Prosthetic hands with realistic human hand-like appearance and warmth can help users naturally integrate into social environments ${ }^{4}$. The outermost skin layer of the e-glove system can incorporate human skin tones, textures, artificial nails, and other features. Figure 4a shows representative examples of the e-glove systems colored with a range of commercial pigments (Slic $\mathrm{Pig}^{\mathrm{TM}}$, Flesh tone silicone pigment, Smooth-On, USA), wherein a detailed surface texture is obtained by exploiting a molding technique (see the Methods section for the details). Enlarged views of textured fingerprints (top) and an artificial nail (bottom) highlight the details of these features. Representative system-level demonstrations of the e-glove systems in several circumstances envisioned such as shaking a hand, tapping a ball, touching a wet diaper, and holding a cup of hot water appear in Supplementary Movies S2-S5, respectively.

To replicate human hand-like warmth, a stretchable Joule-heating system is incorporated under the outermost skin layer of the e-glove system. The basic electronic components of this system include (1) serpentine resistive patterns of a flexible Ag paste for the Joule-heating element, which is stretchable up to $~ 40 \%$ strain without any degradation in the performance (Fig. 4b), (2) a microcontroller unit that is capable of maintaining consistent temperature by exploiting the embedded proportionalintegral-derivative (PID, $P=2, I=5$ and $D=0$ ) (Fig. 4c), and (3) an automatic shutdown unit to eliminate potential overheating risk by which an immediate shutdown of the entire system occurs when any inadvertent incident of overheating beyond the preset limit $\left(40^{\circ} \mathrm{C}\right)$ is detected (Fig. 4d). The basic circuit configuration of the internal 


\section{a}
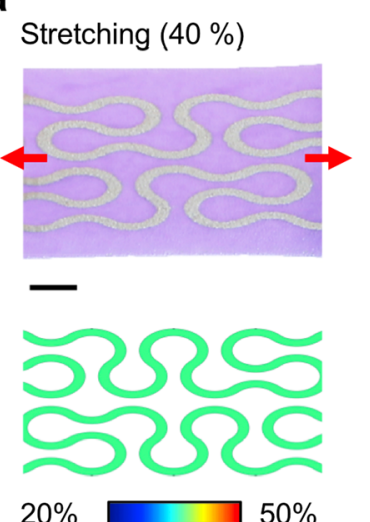

$20 \%$ b
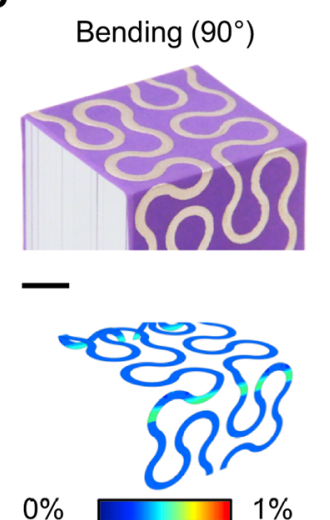

C

Folding $\left(180^{\circ}\right)$
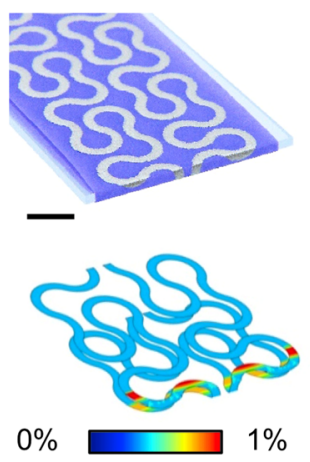

d
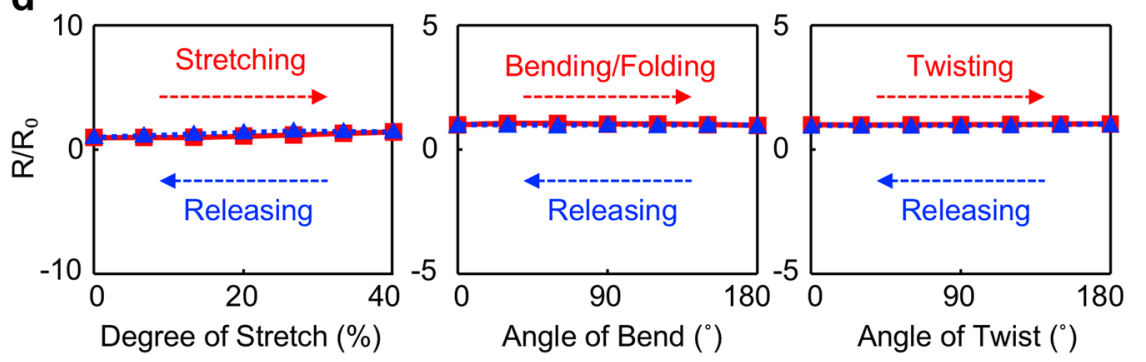

Fig. 3 Mechanical behaviors of replicating human skin-like properties. a Experimental and finite element analysis (FEA) results for a representative testbed unit under stretching at $40 \%$ strain. Scale bar is $7 \mathrm{~mm}$. b Results for the testbed unit under bending at $90^{\circ}$. Scale bar is $5 \mathrm{~mm}$. $\mathbf{c}$ Results for the testbed unit under folding at $180^{\circ}$. Scale bar is $6 \mathrm{~mm}$. $\mathbf{d}$ Experimental data of normalized relative resistance $\left(R / R_{0}\right)$ curves under stretching up to $40 \%$ strain and release back to $0 \%$ (left), bending to $180^{\circ}$ and back to $0^{\circ}$ (middle) and twisting to $180^{\circ}$ and back to $0^{\circ}$ (right)

electronics appears in Supplementary Fig. S10a, wherein a miniaturized $\mathrm{p}$-i-n Si diode-based temperature sensor chip (RN142ZS, p-i-n diode, $0.6 \mathrm{~mm} \times 0.3 \mathrm{~mm}$, Rohm Semiconductor, Japan, sensitivity: $\sim 2.24 \mathrm{mV} /{ }^{\circ} \mathrm{C}$ ) is added to this shutdown unit to detect overheating events (Supplementary Fig. S10b). In this scheme, the trigger of controlled heat (warmth) occurs by pushing a button on the control wristwatch unit in an on-demand manner (Supplementary Movie S6) whenever necessary (i.e., before shaking a hand), while the resistive-based temperature sensors at the center of the palm remain deactivated. Figure 4e shows the experimental (IR, left) and computational (FEA, right) results of the warmed e-glove system in which the skin temperature remains at a preset temperature of $\sim 35^{\circ} \mathrm{C}$. The exploded view (Fig. 4f) of the FEA results reveals the temperature distribution of several selected layers of the e-glove system, implying that the prosthetic hand experiences similar or slightly lower temperature than that of the outer skin layer $\left(\sim 35^{\circ} \mathrm{C}\right)$ due to reduction of the temperature through the adhesive layer (i.e., the epoxy) and the substrate (i.e., the nitrile glove), which have low thermal conductivities of 0.1 and $0.24 \mathrm{~W} / \mathrm{mK}$, respectively.
Experimental demonstrations of the e-glove systems in interactions with human subjects provide assessments of how well the systems replicate the details of a real human hand; a close resemblance to a real hand can enhance the confidence and ability of the prosthetic hand user in many social interactions. Figure 5a presents a within-subjects experimental design that includes four different prototypes featured with human hand-like softness and skin tone (A), along with textures (B), warmth (C), and texture and warmth (D), all deployed in a randomized sequence to eliminate learning bias. A total of 32 subjects, including 24 males and eight females, with an average age of 30 were recruited for this study. Seventeen of the subjects had seen or interacted with the prosthetic hand before the tests. The subjects were asked to interact with each of the prototypes sequentially by touching, poking, scratching/ rubbing, and handshaking gently or firmly (Fig. 5b). Subsequently, the subjects were asked to complete a questionnaire consisting of 12 questions totaling 60 points with ratings on a scale from 1 (low) to 5 (high) to evaluate the comfort, warmth, convenience, and human-like feeling after every interaction (Supplementary Fig. S11), and finally, rank the prototypes in a comparative evaluation. 
a
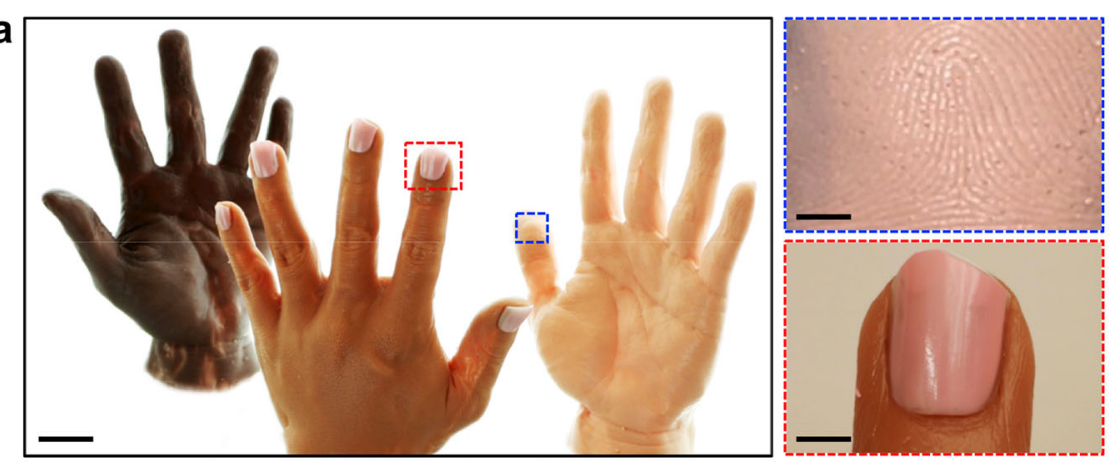

b
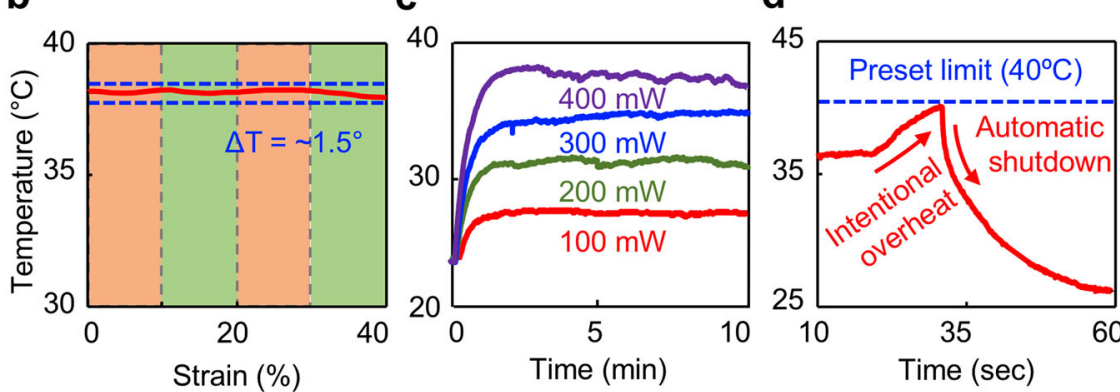

e

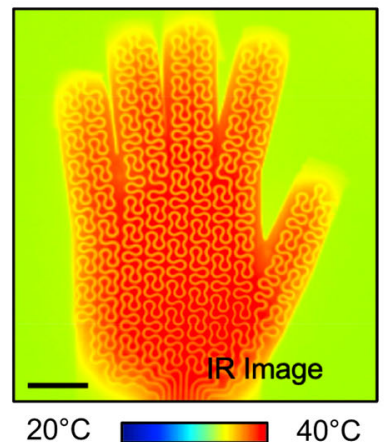

f
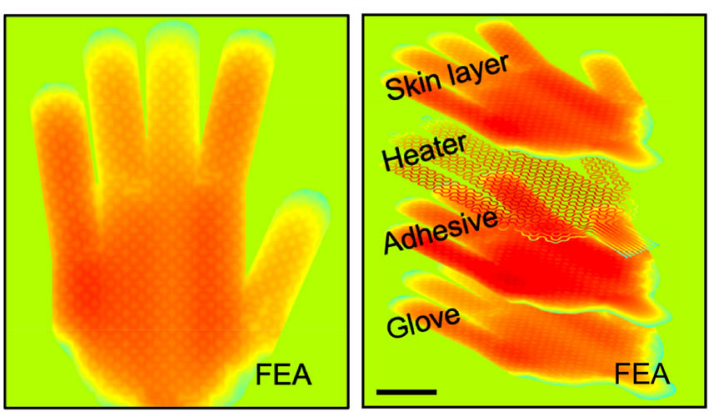

Fig. 4 Demonstration of human hand-like appearance and warmth. a Optical image of the e-glove systems featured with several different skin tones, textures, and nails. The enlarged images highlight the detailed features. Scale bars are $2.5 \mathrm{~cm}$ (left), $5 \mathrm{~mm}$ (right top) and $6 \mathrm{~mm}$ (right bottom), respectively. $\mathbf{b}$ Temperature measured for the warmed skin of the e-glove system under stretching up to $40 \%$ strain. $\mathbf{c}$ Temperature measured over time by increasing the applied power from $100 \mathrm{~mW}$ to $400 \mathrm{~mW}$. d Demonstration of the embedded automatic shutdown upon an intended incident of overheating beyond the preset temperature of $40^{\circ} \mathrm{C}$. e IR image (left) and FEA results (right) for the warmed skin of the e-glove system maintained at $\sim 35^{\circ} \mathrm{C}$. Scale bar is $2.5 \mathrm{~cm}$. f FEA results of the temperature distributions at several selected layers of the e-glove system. Scale bar is $3 \mathrm{~cm}$

The average duration of a subject study was $\sim 40 \mathrm{~min}$, and no skin irritations or adverse effects to the subjects' hands were observed throughout the studies.

Figure $5 \mathrm{c}$ presents the results of one-way repeated measures analysis of variance (ANOVA) test ${ }^{23}$, indicating a substantial difference $(F(3,93)=17.94, p<0.00001)$ at $p \leq$ 0.05 in the subjects' preference to the e-glove prototypes with human hand-like features. Mauchly's test for sphericity $\left(X^{2}(5)=2.79, p=0.73\right)$ confirms that no violation on the sphericity (univariate) assumption exists ${ }^{24}$. The results of post hoc tests using the two-tailed paired samples $t$-test on the dependent means ${ }^{25}$ reveal that prototypes B-D with at least one human hand-like feature (either texture, warmth or both) yield significantly higher rating scores compared with the counterpart (A-B: $t(31)=4.99, p<0.00005$; A-C: $t$ $(31)=3.19, p<0.00324 ; \mathrm{A}-\mathrm{D}: t(31)=6.21, p<0.00001)$. While there is no significant difference between prototypes $\mathrm{B}$ and $\mathrm{D}(t(31)=1.53, p=0.13638)$, there are significant differences between $\mathrm{B}$ and $\mathrm{C}(t(31)=-2.40, p=0.02246)$ and $\mathrm{D}$ and $\mathrm{C}(t(31)=4.16, p=0.00024)$. The corresponding summary table appears in Supplementary Table S3. The results appearing in Fig. 5d, e support that prototype D is the most preferred (ranked) while prototype $C$ is not preferred over prototypes B and D. 
a
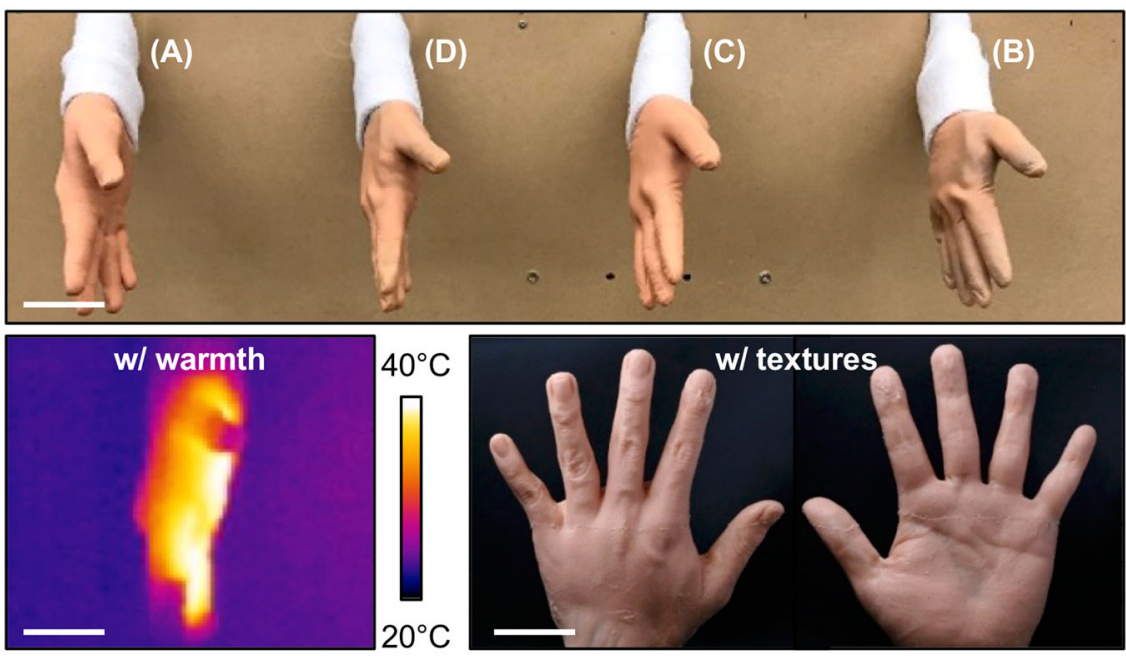

b

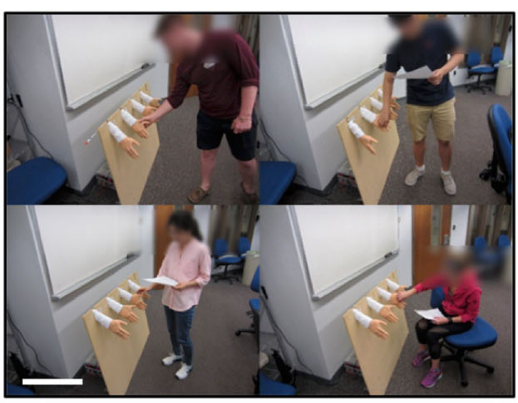

d

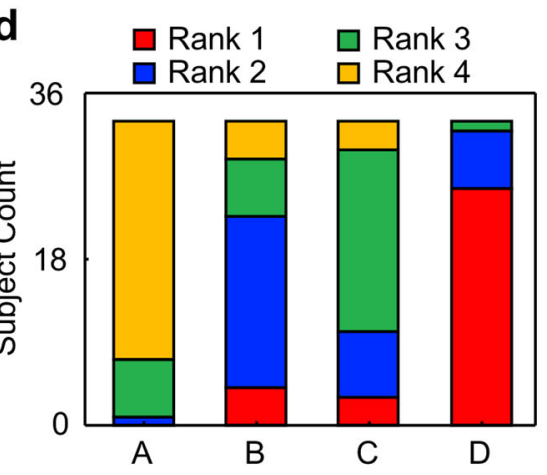

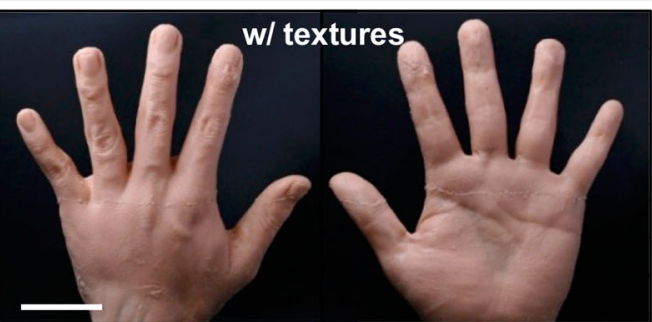

C

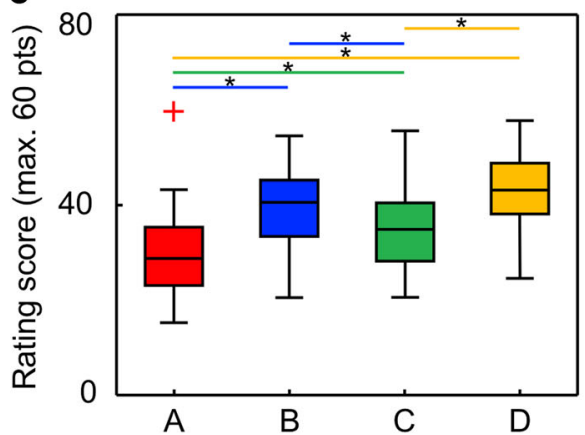

e

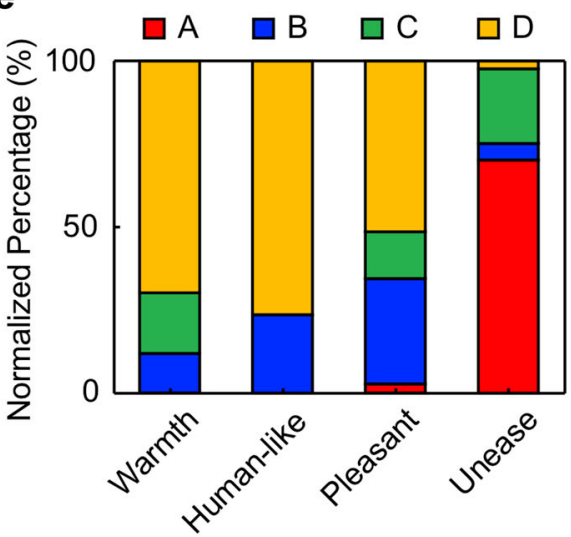

Fig. 5 Assessment of prosthetic hand-human interaction scenarios. a Optical image (top frame) of the experimental setup for the four different prototypes. The IR and optical images (bottom frame) show the human hand-like warmed and textured e-glove prototypes, respectively. Scale bars are $7 \mathrm{~cm}$ (top), $6 \mathrm{~cm}$ (left bottom), and $5 \mathrm{~cm}$ (right bottom), respectively. b Optical images of the participants interacting with the prototypes. Scale bar is $60 \mathrm{~cm}$. c Statistical analysis results of the subject rating score, one-way ANOVA with two-tailed paired sample $t$-test post hoc test in the humanhand interaction study. $\mathbf{d}$ Results of subject responses on the prototypes A-D, ranked from 1 (best) to 4 (worst). e Results of the subject ranking of the prototypes as normalized percentages of the categories of warmth, human-like, pleasant, and unease

\section{Discussion}

The results presented here demonstrate that various electronic circuits and sensors can be printed on a commercial stretchable nitrile glove that already possesses the desired ergonomic design, allowing for seamless integration with arbitrary hand shapes. This integration has been challenging using conventional approaches that typically involve the use of multiple flexible sensors wrapped around prosthetic hands to accommodate the geometric complexity of the hand shape ${ }^{7}$. The user 
interface via a wristwatch unit provides the advantages of the capability for real-time display of the sensory data measured on a control wristwatch unit, remote data transmission to an external reader for data postprocessing, providing benefits and convenience to the user, and a multisensory feedback/display in one device (e.g., temperature, pressure, and humidity sensing two-dimensional data of the whole palm). Adding additional sensory cues through audio and tactile/vibrational feedback to further improve the user interface would be interesting ${ }^{26}$. The realistic human hand-like features of the e-glove system offer an expanded set of options for the daily activities of prosthetic hand users, with the potential to improve their mental health and well-being by helping the user more naturally integrate into social contexts. Although this study focuses on applications for general passive prosthetic hands, the results also suggest opportunities in the integration of the e-glove system with the recently emerging cutting-edge active prosthetic hands controlled by the mind, voice, and/or muscles of the user ${ }^{27-30}$. The hybrid printing method for the fabrication of the e-glove system is cost-effective and compatible with various electronic materials and sophisticated design layouts, thereby foreshadowing the implications for a wide range of users; further development of an e-glove system that can fit the hand of a small child or an extra-large adult is also important. Finally, the application of the established e-glove platform can also be extended to smart gloves for assistive robotic hands, automotive factory workers, and home-based rehabilitations ${ }^{31}$.

\section{Acknowledgements}

C.H.L acknowledges funding support from the Eli Lilly and Company (F.00120802.02.013) and the Purdue Research Foundation (PRF).

\section{Author details \\ 'Weldon School of Biomedical Engineering, Purdue University, West Lafayette, IN 47907, USA. ${ }^{2}$ Department of Computer and Information Technology, Purdue University, West Lafayette, IN 47907, USA. ${ }^{3}$ Department of Computer Science, University of Georgia, Athens, GA 30602, USA. ${ }^{4}$ Department of Aerospace Engineering and Engineering Mechanics, University of Texas at Austin, Austin, TX 78712, USA. ${ }^{5}$ School of Mechanical Engineering, Purdue University, West Lafayette, IN 47907, USA. ${ }^{6}$ Department of Speech, Language and Hearing Sciences, Purdue University, West Lafayette, IN 47907, USA}

\section{Authors' contributions}

C.H.L. devised the concept of the e-glove system. M.K.K., R.N.P., L.W., N.L., B.C.M., C.H.L. designed the research. M.K.K., Y.P., B.K., S.J.L., C.H.L. developed and implemented the e-glove system. R.N.P, B.C.M. performed the validation studies on human-prosthetic hand interactions. L.W., N.L. performed the modeling calculations. M.K.K., R.N.P., L.W., N.L., B.C.M., C.H.L. wrote the paper. All authors reviewed and commented on the paper

\section{Data availability}

The authors declare that all data supporting the findings of this study are available within the paper and its supplementary information.

\section{Conflict of interest}

Purdue University has filed a provisional patent application related to this technology.
Publisher's note

Springer Nature remains neutral with regard to jurisdictional claims in published maps and institutional affiliations.

Supplementary information is available for this paper at https://doi.org/ 10.1038/s41427-019-0143-9.

Received: 26 February 2019 Revised: 5 May 2019 Accepted: 21 May 2019. Published online: 30 August 2019

\section{References}

1. Mckechnie, P. \& John, A. Anxiety and depression following traumatic limb amputation: A systematic review. Inj.-Int. J. Care Inj. 45, 1859-1866 (2014).

2. Grob, M., Papadopulos, N. A., Zimmermann, A., Biemer, E. \& Kovacs, L. The psychological impact of severe hand injury. J. Hand Surg.-Eur. 33, 358-362 (2008).

3. Gallagher, P. \& Desmond, D. Measuring quality of life in prosthetic practice: benefits and challenges. Prosthet. Orthot. Int. 31, 167-176 (2007).

4. Cabibihan, J., Pattofatto, S., Jomaa, M., Benallal, A. \& Carrozza, M. Towards humanlike social touch for sociable robotics and prosthetics: comparisons on the compliance, conformance and hysteresis of synthetic and human fingertip skins. Int. J. Soc. Robot. 1, 29-40 (2009).

5. Cordella, F. et al. Literature review on needs of upper limb prosthesis users. Front. Neurosci. 10, 209 (2016).

6. Kim, D. et al. Epidermal electronics. Science 333, 838-843 (2011).

7. Kim, J. et al. Stretchable silicon nanoribbon electronics for skin prosthesis. Nat. Commun. 5, 5747 (2014)

8. Wang, S. et al. Skin electronics from scalable fabrication of an intrinsically stretchable transistor array. Nature 555, 83-88 (2018).

9. Kaltenbrunner, M. et al. An ultra-lightweight design for imperceptible plastic electronics. Nature 499, 458-463 (2013).

10. Chossat, J.-B., Shin, H.-S., Park, Y.-L. \& Duchaine, V. Soft Tactile skin using an embedded ionic liquid and tomographic imaging. J. Mech. Robotics-Trans. Asme 7, 021008 (2015)

11. Chortos, A., Liu, J. \& Bao, Z. A. Pursuing prosthetic electronic skin. Nat. Mater 15, 937-950 (2016).

12. Ota, $\mathrm{H}$. et al. Application of $3 \mathrm{D}$ printing for smart objects with embedded electronic sensors and systems. Adv. Mater. Technol. 1, 1600013 (2016).

13. Mishra, R. K. et al. Wearable flexible and stretchable glove biosensor for on-site detection of organophosphorus chemical threats. ACS Sensors 2, 553-561 (2017).

14. Boley, J. W., White, E. L. \& Kramer, R. K. Mechanically sintered gallium-indium nanoparticles. Adv. Mater. 27, 2355-2360 (2015).

15. Lee, P. et al. Highly stretchable and highly conductive metal electrode by very long metal nanowire percolation network. Adv. Mater. 24, 3326-3332 (2012).

16. Carlson, A., Bowen, A., Huang, Y., Nuzzo, R. \& Rogers, J. Transfer printing techniques for materials assembly and micro/nanodevice fabrication. Adv. Mater. 24, 5284-5318 (2012).

17. Kim, S. et al. Microstructured elastomeric surfaces with reversible adhesion and examples of their use in deterministic assembly by transfer printing. Proc. Natl Acad. Sci. USA 107, 17095-17100 (2010).

18. Wie, D. S. et al. Wafer-recyclable, environment-friendly transfer printing for large-scale thin-film nanoelectronics. Proc. Natl Acad. Sci. USA 115, 7236-7244 (2018).

19. Lee, C. et al. Soft core/shell packages for stretchable electronics. Adv. Funct. Mater. 25, 3698-3704 (2015).

20. Heikenfeld, J. et al. Wearable sensors: modalities, challenges, and prospects Lab a Chip 18, 217-248 (2018)

21. Han, S. et al. Mechanically reinforced skin-electronics with networked nanocomposite elastomer. Adv. Mater. 28, 10257-10265 (2016).

22. Kim, D. H. et al. Optimized structural designs for stretchable silicon integrated circuits. Small 5, 2841-2847 (2009).

23. Girden, E. R. ANOVA: Repeated measures, Vol. 84. (Sage Publications, Inc. Thousand Oaks, CA, USA, 1992).

24. Mauchly, J. W. Significance test for sphericity of a normal n-variate distribution Ann. Math. Stat. 11, 204-209 (1940)

25. Norušis, M. J. SPSS 14.0 guide to data analysis. (Prentice Hall, Upper Saddle River, NJ, USA, 2006) 
26. Cipriani, $C$. et al. A novel concept for a prosthetic hand with a bidirectional interface: A Feasibility Study. IEEE Trans. Biomed. Eng. 56, 2739-2743 (2009).

27. Moran, C. W. Revolutionizing prosthetics 2009 modular prosthetic limb-body interface: overview of the prosthetic socket development. Johns. Hopkins Apl. Tech. Dig. 30, 240-249 (2011).

28. Hutchinson, D. T. The quest for the bionic arm. J. Am. Acad. Orthop. Surg. 22 346-351 (2014).
29. Raspopovic, S. et al. Restoring natural sensory feedback in real-time bidirectional hand prostheses. Sci. Trans. Med. 6, 222ra19 (2014).

30. Tabot, G. A. et al. Restoring the sense of touch with a prosthetic hand through a brain interface. Proc. Natl Acad. Sci. USA 110, 18279-18284 (2013).

31. Gurari, N., Kuchenbecker, K. J. \& Okamura, A. M. Perception of springs with visual and proprioceptive motion cues: implications for prosthetics. IEEE Trans. Hum.-Mach. Syst. 43, 102-114 (2013). 